\title{
Simulated Annealing Optimization Bat Algorithm in Service Migration Joining the Gauss Perturbation
}

\author{
Zhao Guodong ${ }^{1}$, Zhou Ying ${ }^{2}$ and Song Liya ${ }^{3}$ \\ ${ }^{1}$ School of Mathematics and Computer Science, Ningxia University, Yinchuan \\ 750021, China \\ ${ }^{2}$ School of Science and Engineering, Tianjin Open University 300387, China \\ ${ }^{3}$ Department of physics and Electrical Engineering, Ningxia University, Yinchuan, \\ 750021, China \\ guodonzho@163.com
}

\begin{abstract}
Bat algorithm is an optimization method inspired by the echo-location bats to search in nature, hunt prey behavior, combining multi-agent system and evolution mechanism. To improve the search results of BA algorithm, this paper proposes a gauss perturbation bats optimization algorithm based on simulated annealing (SAGBA). Firstly, the bionic principle, optimization mechanism and characteristics of the bat algorithm are analyzed and the algorithm optimization process are defined; Then the idea of the simulated annealing is put into bat optimization algorithm, and Gaussian disturbance is carried out to some individuals using the bat algorithm and strengthen the ability of the bat algorithm jumping out of local optimal solution. Finally, conduct simulations are respectively compared in 20 typical benchmark test functions among bat optimization algorithm, simulates annealing particle swarm algorithm and SAGBA algorithm. The results show that SAGBA algorithm not only increases the global convergence, but convergence speed and accuracy are better than other two algorithms.
\end{abstract}

Keywords: Bat algorithm, Simulated annealing, Gauss perturbation, The simulation, Optimization

\section{Introduction}

Most decisions in the field of engineering technology, economic management, and other problems can be summed up in optimization problem; its quality decides strengthens and weakness of decision scheme [1]. Traditional optimization methods such as linear programming, dynamic programming, etc., or the optimization problem with constraints (such as the objective function is continuous and differentiable), or because the attributes of optimization problem (e.g., NP problem) can only solve the small problem, for large, strong constraints, the nonlinear such optimization problems is difficult to solve, or the effect not good $[2,3]$. Swarm intelligence algorithm is a new evolutionary computation technique developed in recent years, is easy to implement, suitable for parallel processing, strong robustness, etc., [4], the typical algorithms such as ant colony algorithm and particle swarm optimization algorithm, the former is the bionic simulation of ant colony foraging behavior, the latter is for the birds feeding behavior reflects the simple simulation of the social system, both in the field of continuous optimization and combinatorial optimization be widely applied.

Intelligent optimization methods inspired and evolved by nature rules and biological swarm intelligence behavior, such as genetic algorithm, ant colony algorithm, particle 
swarm optimization algorithm, etc., in the traditional optimization technology area, show the good performance. In recent years, scholars has presented some novel bionic swarm intelligence algorithm, such as fish algorithm [1], the swarm algorithm [5], the firefly algorithm $(4,5)$, etc., in scientific computing and engineering field shows unique characteristics and application effect, increasingly concerned by academia and engineering. artificial fish [6] by constructing fish algorithm is used to imitate foraging behavior, poly group of the fish, train to search for most nutrient-rich waters so as to realize optimization; Colony algorithm $[7,8]$ is a evolved optimization technique from mimic bees behavior, bees, use different search pattern according to the division in labor, through the communication between different roles, conversion, and cooperation to realize the bees swarm intelligence, and then complete the optimization work; Firefly algorithm [9] is a constructed class of stochastic optimization algorithm by simulating the firefly bioluminescent behavior in the nature, using fluorescence characteristics of the species endemic to looking for a partner, according to the search area and has a better location within the neighborhood structure of the individual to achieve mobile location evolution. Swarm intelligence algorithm in the process of solving problems, the set of all possible solutions are generally considered as a solution space, starting from a subset solutions of the representative problem, by applying the operator to a subset to get the new solution set, and gradually make the population evolve to the optimum solution included or approximate optimal solution of the state, and only need the objective function information in the process of evolution, can find the optimal solution without being limited by the continuous and differentiable search space.

As a result, they are widely used in parameter optimization [10], data [11] and image processing [12], production scheduling path planning [13] and the social sciences [14], and other fields, and to get the attention of many researchers.

Bats rely on echo-location to hunt bats, usually in search of prey from about 10 to 20 per second, sound intensity up to 110 decibels of ultrasonic pulse; pulse sound intensity is usually in search of prey, the largest decreases when flying to the prey, pulse frequency increased at the same time, about 200 pulses per second launch. Pulse powerful sound help ultrasonic spread to farther distance, pulse frequency high help accurately master the changing spatial location. Bats produce echolocation sound waves in general by FM signal of single or multiple harmonic wideband, frequency are usually in $25 \mathrm{Kz}-100 \mathrm{Kz}$ range, each harmonic frequency from high to low, falling faster, more harmonic, wideband FM sound fine structure to determine the complex environment and its prey. If the bats in the nature simulation for the multi-agent distribution in the search space, to measure individual strengths and weaknesses of the location using optimization objective function values, the bats search target and fly move process simulation for good feasible solution to replace poor feasible solution of the update process, bats use ultrasonic to detect, locate, hunt prey and a series of behavior can use the following equation to simulate [15]. Bats can avoid obstacles of the hair thickness and hunt prey in a completely dark environment, thanks to have amazing echo-location in bats. Bat not only can detect the distance of the prey, and to identify its bodily form feature, location and Angle by detecting the ultrasonic echo time delay, using the echoes to the binaural time difference, the change of the sound intensity establish the environment of the three-dimensional scene. Bats accurate location in complex environment, catching and algorithm are similar to the optimization of high-dimensional complex space condition, for the simulation of the former biological mechanism is optimize brought inspired. Bats algorithm is a kind of random global optimization algorithm comes developed from the ultrasonic search in the nature and the biological characteristics of hunting prey, the first put forward by Yang X.S. [16], the bat algorithm [17] (bat algorithm (BA)) is heuristic search algorithm proposed by Xin - She Yang in 2010 based on swarm intelligence, is a kind of effective method to search the global optimal solution. 
The algorithm is a kind of technique based on iterative optimization, initialized to a group of random solutions, and then through the iterative search for the optimal solution, and the optimal solution by random flight around local data processing, to strengthen the local search. Compared with other algorithms, the algorithm of BA is far superior to other algorithms in terms of accuracy and effectiveness, and not many parameters to be adjusted. BA algorithm has been used in engineering design [19], classification [20], fuzzy clustering [14], predict [7, 8] and neural network, etc

This paper proposes that the simulated annealing optimization algorithm bat join gaussian disturbance of, innovative mainly as follows:

(a) This paper analyzes the bionic principle of the algorithm, optimization the mechanism and characteristics, proposes a gaussian disturbance bats optimization algorithm based on simulated annealing (SAGBA), the algorithm not only maintains the characteristics of the bats' simple and effective optimization algorithm, and enhances the bat algorithm's ability to jump out of local optimal solution, to improve the convergence speed of the algorithm.

(b) Simulation test conducts for the proposed algorithms' optimization performance in continuous space and discrete space, compares the evolution and the average global optimal value among the SAGBA ,BA and SAPSO curve, and statistical validation for the three algorithms, combinatorial optimization test, verify the algorithm in the function optimization and the feasibility and effectiveness of combinatorial optimization aspects.

\section{Proposed Algorithm}

\section{A. Biological Mechanism and Mathematical Simulation of the Algorithm}

\section{(A) Algorithm steps}

Bats are the only mammals with wings, and they have advanced echo positioning capability [9]. Most of the miniature bats are insectivores' animals. Miniature bats using acoustic echo-location in detecting prey and avoiding obstacles, and finds himself located in the habitat of cracks in the dark. These bats emit a loud voice, and then hear from the surrounding objects reflected echo.

For different bats, their pulse is associated with the strategy of hunting. Most bats through a filter with a short and high frequency signal scan around, while the other bats frequently use echolocation of fixed signals. The signal bandwidth changes depending on the kinds of bats, and often increasing through using more harmonic.

Using the some echo-location characteristics of the ideal micro in bats, the bats idealized rules are as follows

All bats use feel difference of echolocation to judge the difference between food, food and swim around obstacles;

Bats in speed VI, position the xi and fixed frequency $f_{\min }$ (or wavelength $\lambda$ ) random flight, with different wavelength $\lambda$ (or frequency f) and volume A0 to search for prey. They will automatically according to the proximity of prey to adjust their pulse wavelength (or frequency)

Although the volume changes different under different forms, here assumes that the volume from a big A0 (positive) changes to the minimum ${ }_{A_{\min }}$.

Based on the rules of the ideal, the basic steps of the bat algorithm (BA) can be summarized as follows

Initialization bat populations ${ }^{x_{i}}$ and ${ }^{v_{i}} \quad(\mathrm{I}=1,2 \ldots \mathrm{n})$

The initial frequency $f_{i}$, pulse rate ${ }^{r_{i}}$ and volume ${ }^{A_{i}}$

While ( $\mathrm{t} \prec$ maximum number of iterations) 
By adjusting the frequency, generating new solutions, at the same time, according to the formula (1) to (3) to update the velocity and position

If ( (rand $>r_{i}$ )

Select a solution concentration from these optimal solution

In the choice of the near optimal solution generate a local solution

end if

Generate a new by randomly flight

If (rand $\prec_{A_{i}} \& \mathrm{f}\left({ }^{x_{i}}\right) \prec \mathrm{f}\left({ }^{x_{*}}\right)$ )

To accept the new

Increase the value of $r_{i}$ and decrease the value of $A_{i}$

end if

The bat sort, find the optimal solution ${ }^{x}$.

end while

An obvious simplify of the bat algorithm is to estimate time delay and the three dimensional terrain with unlimited tracks. Although its application in the geometry calculation is very good, because we are faced with the most is a multidimensional problem, rarely use it in practice. In addition to these simplifying assumptions, we can also use some approximation. Usually, the range of frequencies $f$ is $\left[f_{\min }, f_{\max }\right]$ corresponding to the wavelength range is $\left[\lambda_{\min }, \lambda_{\max }\right]$. For example, a frequency range is $[20 \mathrm{kHz}, 500 \mathrm{kHz}$ ], corresponding to the range of wavelength is from $0.7 \mathrm{~mm}$ to $17 \mathrm{~mm}$

(B) The movement of virtual bats

In the simulation, we must use the virtual bats. In the searching space of a d, $\mathrm{t}$ moment, in bat algorithm, update the position ${ }^{x_{i}^{\prime}}$ and speed $v_{i}^{\prime}$ of the Bat I according to the following formula

$$
\begin{gathered}
f_{i}=f_{\min }+\left(f_{\max }-f_{\min }\right) \beta \\
v_{i}^{t}=v_{i}^{t-1}+\left(x_{i}^{t-1}-x_{*}\right) f_{i} \\
x_{i}^{t}=x_{i}^{t-1}+v_{i}^{t}
\end{gathered}
$$

$f_{i}$ For frequency, $\beta \in[0,1]$ is a random variable of uniform distribution on, ${ }^{x_{*}}$ for the current global optimal position, it is the optimal position after searching all the bats and getting the position of the comparison. $\lambda_{i} f_{i}$ Is increment speed, we can accord the needs of specific issues, fixed a factor ${ }^{\lambda_{i}}$ (or $f_{i}$ ), at the same time using another factor $f_{i}$ (or ${ }^{\lambda_{i}}$ ) to adjust the speed of change

For local search, once selected a solution in the current optimal solution, so every bat' new solution according to the local data processing for random walk is:

$$
x_{\text {new }}=x_{\text {old }}+\varepsilon A^{t}
$$

Which $=\in[-1,1]$ is a random number; ${ }^{A^{\prime}}=A_{i}^{\prime}$ is the average volume of all the bats in the same period. Bat speed and position update steps some similar to the standard particle swarm optimization [10]. To a certain extent, BA can be regarded as a standard particle swarm optimization is combined with the balance of the strengthening of local search; the balance is controlled by the volume and incidence rate of the pulse

(C) The volume and pulse rate

The volume ${ }^{A}$, and pulse rate ${ }^{r}$. updated according to the following iterative process. When bats locate prey, will reduce the volume, pulse rate will increase at the same time, the volume change in any convenient value. For example, for the sake of simplicity, 
available ${ }^{A_{0}=1, A_{\min }=0}$, assumptions, ${ }^{A_{\min }}=0$ and means that a bat just find their prey and temporarily stop any sound. Update the formula is:

$$
A_{i}^{t+1}=\alpha A_{i}^{t}, \quad r_{i}^{t+1}=r_{i}^{0}[1-\exp (-\gamma t)]
$$

$\alpha$ and $\gamma$ as constants. In fact, $\alpha$ is similar to a cooling factor of the cooling process in the simulated annealing earlier discussed in this book. For ${ }^{0<\alpha<1, \gamma>0}$, we have to arbitrary

$$
A_{i}^{t} \rightarrow 0, \quad r_{i}^{t} \rightarrow r_{i}^{0} \text {, 当 } t \rightarrow \infty
$$

The simplest case is that ${ }^{\alpha}=\gamma$, in the implementation, we use the ${ }^{\alpha}=\gamma=0.9 \square 0.975$

\section{B. Simulated Annealing}

Simulated annealing algorithm (SA) is one of the simplest and most popular heuristic algorithms; it is global random search method based on the trajectory [11]. The SA algorithm is simulated annealing process of material processing, has been widely used in combinatorial optimization problem. SA algorithm has the ability of probabilistic kick in the process of search, can effectively avoid into local extremum in the process of search, it not only accept good solutions in the process of annealing, but also accept difference solution at a certain probability, the probability under temperature parameters control at the same time, with the decrease of temperature drop and the size. In fact, under the enough slow cooling rates, SA will converge to the global optimal solution. From the essence, simulated annealing belongs to search algorithm of Markov chain under the condition of appropriate convergence, the pseudo code is as follows:

1) Initialization temperature $T 0$ and initial solution $x 0$

2) Set the final temperature $\mathrm{Tf}$ and the maximum iterations number $\mathrm{N}$

3) Define cooling table ${ }^{T} \mapsto \alpha T,(0<\alpha<1)$

4) While $(\mathrm{T}>\mathrm{Tf}, \mathrm{n}<\mathrm{N}$ )

5) Random data processing $x_{n+1}=x_{n}+\varepsilon$

6) Calculate $\Delta f=f_{n+1}\left(x_{n+1}\right)-f_{n}\left(x_{n}\right)$

7) When data processing is superior to accept data processing

8) If not to accept the new

9) Generate a random number $r$

10) If $(p=\exp [-\Delta f / T]>r)$ accepts

11) End if

12) Update the optimal solution $x^{*}$ and optimal value $f^{*}$

13) $\mathrm{n}=\mathrm{n}+1$;

14) End while

Although there are many researchers combine simulated annealing algorithm with other optimization algorithm [12-14], have not seen the simulated annealing algorithm combine with the bat optimization algorithm yet.

\section{The Bat Optimization Algorithm Based on Simulated Annealing}

Introduces simulated annealing thought to bats optimization algorithm, propose a gauss perturbation bats optimization algorithm based on simulated annealing (SAGBA), using the gauss perturbation mutation operation further adjust the optimization of the cluster. Its basic implementation process is randomly generated initial population first, random search, set the initial temperature, using the simulated annealing algorithm to get the global optimal replacement value, then according to the position and the velocity update 
formula to produce a set of data processing, and then in a better position to individual gaussian disturbance, to further search.

In each evolution, mutation operations variation according to gauss perturbation, the location after the gaussian disturbance to replace the original position, namely:

$$
X^{t}=X^{t}+a \oplus \varepsilon
$$

Among them ${ }^{\varepsilon}$ and $X^{t}$ is the random matrix of the same order, each of these elements $\varepsilon^{\varepsilon_{i j} \square N(0,1)}, \oplus$ namely the dot product. In order to avoid excessive volatility, a used to adjust the ${ }^{\varepsilon}$ search scope

The whole algorithm can be divided into three parts: the first part is to use simulated annealing algorithm to find the global optimal replacement value, the second part is to use the bat optimization algorithm to find a set of data processing, the third part of the new gaussian disturbance, for further search. The algorithm steps are as follows

A. Random initialization position, velocity, frequency, pulse rate, and the volume of population;

B. Every bat individual fitness evaluation, store the current position and adapt to the value of each individual and the optimal location and adapt to the value of all the individual adaptive value stored in $\mathrm{p}$ best;

C. To determine the initial temperature;

D. Determine the current temperature according to the type of each individual adaptive value

$$
T F\left(x_{i}\right)=\frac{e^{-\left(f\left(x_{i}\right)-f(\text { pbest })\right) / t}}{\sum_{i=1}^{N} e^{-\left(f\left(x_{i}\right)-f(\text { pbest })\right) / t}}
$$

E. Roulette strategy from all bats individuals identify some alternative global optimal value pbest', and then according to the type of update the speed and position of each individual.

$$
\begin{array}{r}
v_{i}^{t}=v_{i}^{t-1}+\left(x_{i}^{t}-\text { pbest }^{\prime}\right) f_{i} \\
x_{i}^{t}=x_{i}^{t-1}+v_{i}^{t}
\end{array}
$$

A. Counting new target value of all the bats, update the optimal position, on a better location for gaussian disturbance, and compared the location before and after the gauss perturbation, find out the optimal location pbest' and the corresponding optimal value;

B. Conducting temperature annealing operation;

C. If meet the stop condition (usually the accuracy or the number of iterations), stop search, output the result, or go to the D.

D. The initial temperature and annealing temperature on the algorithm has a certain influence, generally uses the following early moderate annealing way [15]

$$
\begin{aligned}
t_{k+1} & =\lambda t_{k} \\
t_{0} & =f(\text { p best }) / \ln 5
\end{aligned}
$$

Among them, $\lambda$ is for the cooling coefficient between [0.5,1].

Algorithm flow chart is shown in Figure 1. 


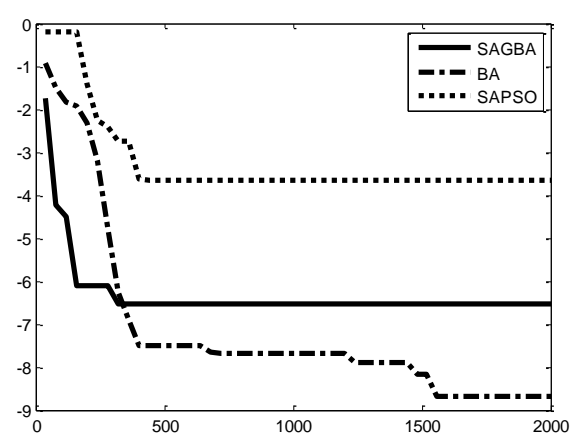

Figure 2. Function Ackley Evolutionary Curve Comparison Chart

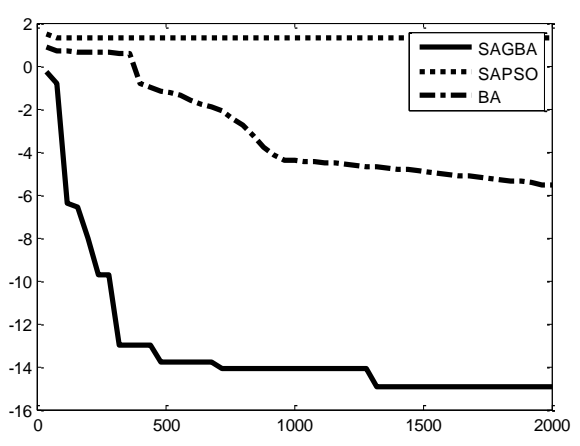

Figure 3. The Evolution Curve Comparison Chart of the Function Beale

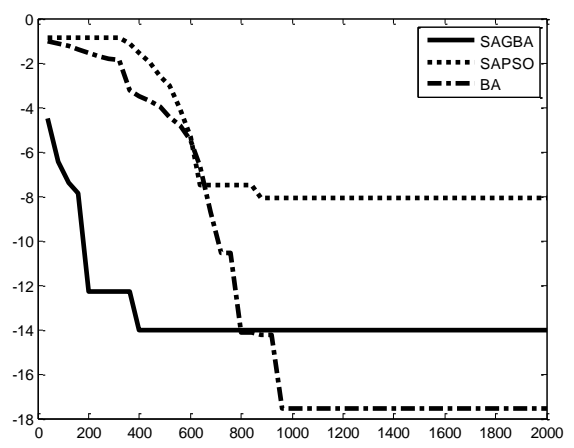

Figure 4. Function Bohachevsky Evolutionary Curve Comparison Chart 


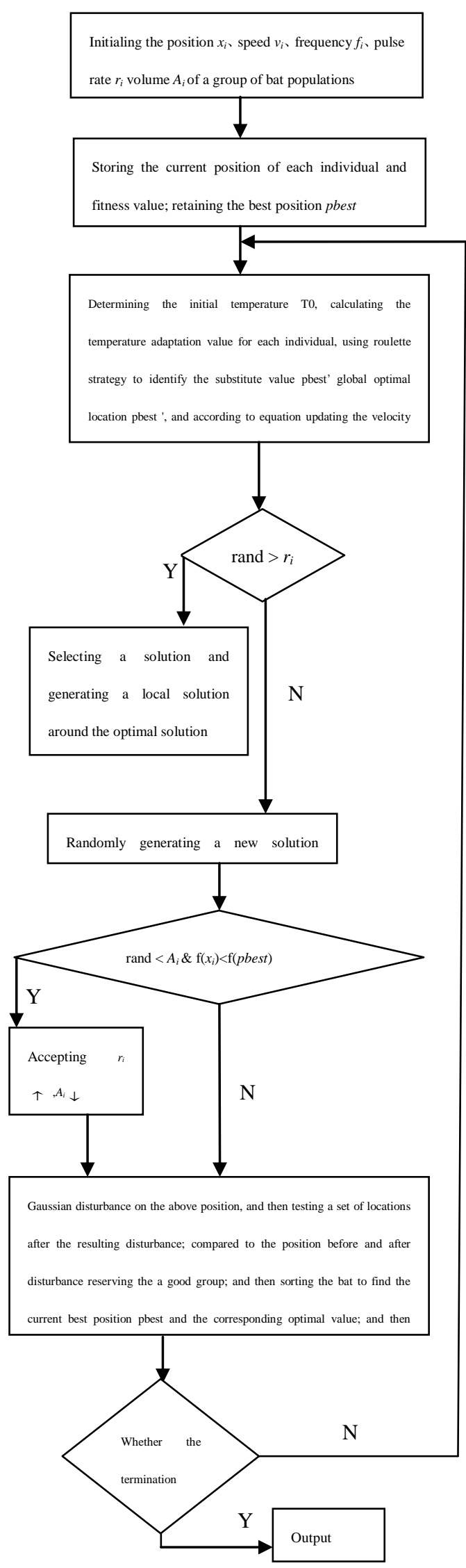

Figure 1. SAGBA Algorithm Flow Chart 


\section{Simulation Experiment}

In order to study the effectiveness of the SAGBA algorithm, we had optimization performance comparison of BA, SAPSO [14] and SAGBA in 20 typical benchmark test functions, can visually see SAGBA algorithm better optimization performance.

\section{A. Experiment Design}

Parameter design of three kinds of algorithm is as shown in Table 1

Table 1. Parameters Design Table

\begin{tabular}{|c|c|}
\hline $\begin{array}{l}\text { algorithm } \\
\text { name }\end{array}$ & Parameter design \\
\hline $\mathrm{BA}$ & The bat number is $40, \alpha=\gamma=0.9$ \\
\hline SAPSO & $\begin{array}{l}\text { Particle number is } 40 \text {; the learning factor is } \mathrm{c} 1=\mathrm{c} 2=2.05 \text {; annealing } \\
\text { constant is } 0.5\end{array}$ \\
\hline SAGBA & Number of bat is 40 ; annealing constant is 0.5 \\
\hline
\end{tabular}

Used in this article's 20 typical benchmark test functions which are from global optimization test function library [16], where "-" means there are many different optimal point, as shown in Table 2.

Table 2. Benchmark Function Table

\begin{tabular}{lcc}
\hline Benchmark functions & The most advantage & optimal value \\
\hline Ackley & $(0,0)$ & 0 \\
Beale & $(3,0.5)$ & 0 \\
Bohachevsky & $(0,0)$ & 0 \\
Booth & $(1,3)$ & 0 \\
Branin & $(9.42478,2.475)$ & 0.3979 \\
Dixon\&Price & $(1, \ldots, 1)$ & 0 \\
Goldstein\&Price & $(0,1)$ & 3 \\
Griewank & $(0,0)$ & 0 \\
Hartmann & $(0.1146,0.5556,0.8525)$ & -3.8628 \\
Hump & $(0.0898,-0.7126)$ & 0 \\
Levy & $(1,1)$ & 0 \\
Matyas & $(0,0)$ & 0 \\
Powell(d=24) & $(3,-1,0,1, \ldots)$ & 0 \\
Rastrigin & $(0,0)$ & 0 \\
Rosenbrock & $(1,1)$ & 0 \\
Shubert & -- & -186.731 \\
Sphere(d=30) & $(0, \ldots, 0)$ & 0 \\
Sum Squares $(d=20)$ & $(0, \ldots, 0)$ & 0 \\
Trid(d=6) & -- & -50 \\
Zakharov & $(0,0)$ & 0 \\
\hline
\end{tabular}

To evaluate the convergence of the algorithm, request the largest number of iterations is set to 2000, continuous running50 times, the average value of the global minimum of test functions as algorithm to optimize the performance of the measure, and the $\mathrm{t}$ - test method [17] comparative analysis on the performance of the algorithm. 


\section{B. Analysis of Experimental Results}

\section{(A) Experiment 1: Evolution Curve Contrast among SAGBA, BA and SAPSO}

Under the same maximum number of iterations, for more than 20 test function to carry on the simulation, as shown in Figure 2 to Figure 21. The following evolution curve evolution, transverse represents average fitness value of the vertical axis represent logarithmic (i.e., the log (fitness value)).

As can be seen from the Figure 2, for Ackley function, at the beginning of the evolutionary, individual quality of the SAGBA algorithm is obviously better than the SAPSO algorithm and BA algorithm. With the increasing number of iterations, SAPSO algorithm already into premature convergence and can't jump out after 400 iterations. BA algorithm compared with SAPSO algorithm in terms of its individual quality is improved, but it also faces into the plight of premature convergence, although in 400 times, 640 times, 1200 inferior multiple occurrences of a turning point, jump out of local optimum, and the convergence precision is higher, its convergence is slow, the number of iterations is relatively large. Although SAGBA algorithm also appeared the phenomenon of premature convergence in the process of iteration, but in the 310th iteration when there is an obvious turning point, after (10-7) to achieve high convergence precision and less number of iterations (310) cases, to achieve the optimal solution, show that the algorithm is to guide the already fall into local optimal value individual deviates from the original local optimal point, with larger probability to be near global optimal point.

Can also be seen in Figure 3, for Beale function, at the beginning of the evolutionary, individual quality of the SAGBA algorithm is obviously better than SAPSO algorithm and BA. With the increasing number of iterations, SAPSO algorithm already into premature convergence and can't jump out after 100 iterations, and did not converge to global optimal point. BA algorithm compared with SAPSO algorithm in terms of its individual quality is improved, but it also faces into the plight of premature convergence, although appeared inflection point at the 800th iteration, jump out of local optimal point, but the convergence accuracy and the number of iterations is not significant. Although SAGBA algorithm also appeared in the process of iteration the phenomenon of premature convergence, but in the 500th iteration is convergent to the global optimal point and reached a very high convergence precision.

From Figure 4, similar to Figure 2, for Bohachevsky function, at the beginning of the evolutionary, the SAGBA algorithm individual quality is obviously better than SAPSO algorithm and BA. With the increasing number of iterations, while SAPSO, BA and SAGBA algorithm are at an inflection point jump out of local optimal point, with larger probability to be near global optimal point, but when the same number of iterations (400th), SAGBA algorithm's convergence speed is faster, and convergence precision is relatively high.

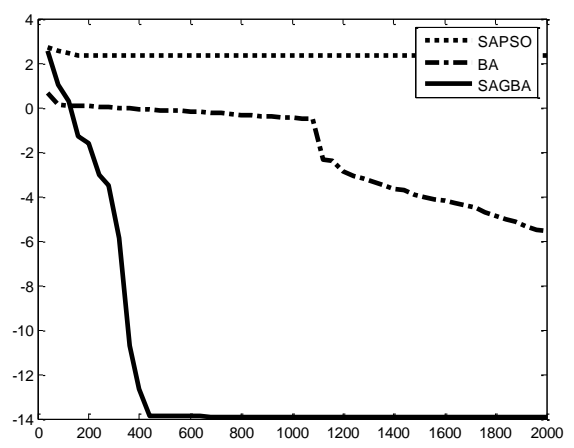

Figure 5. The Evolution Curve Comparison Chart of the Function Booth 
From Figure 5, we can see more easily, for Booth function, only SAGBA algorithm search function to the global optimal point, SAPSO algorithm trapped in local optimal point, and BA algorithm did not achieve convergence state.

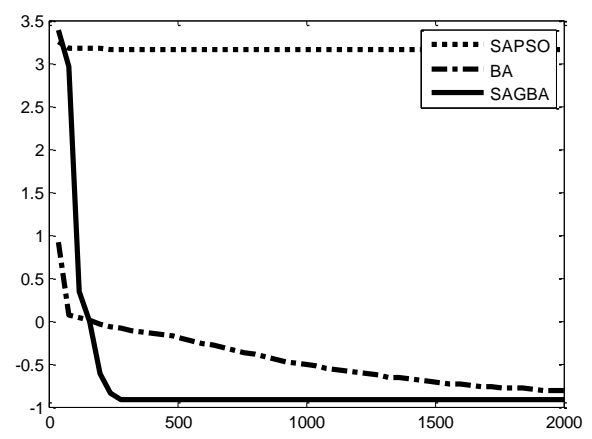

Figure 6. Function Branin Evolutionary Curve Comparison Chart

From Figure 6, for Branin function, although in the early evolution of individual quality of BA algorithm is superior to SAGBA and SAPSO algorithm, SAPSO algorithm trapped in local optimal point and can't jump out, BA algorithm didn't search the global optimal point, only SAGBA algorithm is convergent to the global optimal point.

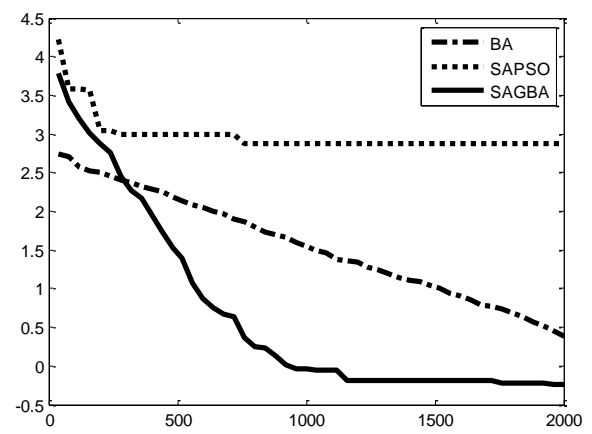

\section{Figure 7. The Evolution Curve Comparison Chart of the Dixon \& Price Function Dixon \& Price}

As can be seen from the Figure 7, for Dixon \& Price function, search ability of three algorithms is similar to Figure 5, Figure 6, only SAGBA algorithm is convergent to the global optimal point, SAPSO algorithm trapped in local optimal point and can't jump out, and BA algorithm didn't search the global optimal point. Throughout all of the function evolution curve contrast figure, can get the following conclusion: SAGBA algorithm than the other two kinds of algorithm convergence speed, better search accuracy, namely convergence of the gauss perturbation bat optimization algorithm based on simulated annealing is superior to convergence of the other two algorithm.

\section{(B) Experiment 2: SAGBA, BA and SAPSO Average Global Optimal Value Contrast}

In order to further study SAGBA optimize performance, under the same experimental conditions, set the maximum number of iterations for 2000 times, the independent running 50 times, take the average of 50 times, where "-" means in the maximum number of iterations for 2000 times is not found in the experiments of 50 times independent minimum, in order to make the chart clearly, now put Boh represents Bohachevsky 
function, Dixon represents Dixon \& Price function, Goldstein represents Goldstein \& Price functions, such as Table 3.

Table 3. Average Optimal Value Contrast Table of the Four Global Algorithms

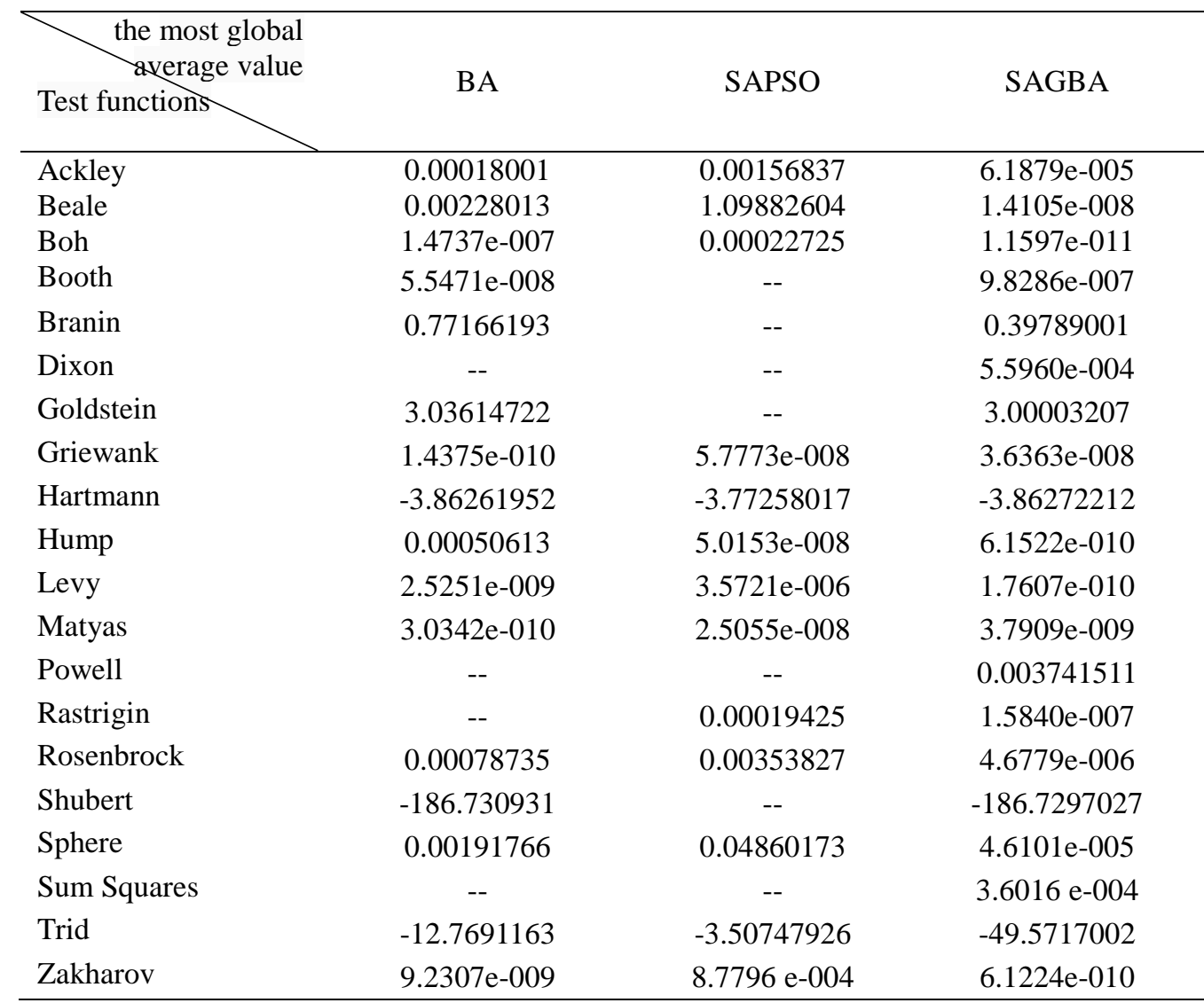

BA algorithm, SAPSO algorithm and SAGBA algorithm results in 20 test function is presented in table 3. Results can be seen from the in the table, the function on the Booth and Matyas, BA algorithm result is better than the SAGBA results, but the result is better than that of SAPSO algorithm; On Griewank function, SAGBA algorithm results to the result of the difference in BA algorithm, and SAPSO algorithm results are about the same; In function Goldstein\& Price, Hartmann and Shubert, SAGBA algorithm results with BA algorithm results are about the same, for besides function, SAGBA algorithm with the calculation results are superior to BA and SAPSO algorithm. Search ability of the gauss perturbation bat optimization algorithm based on simulated annealing is superior to other two kinds of optimization algorithm search ability.

\section{(C) Experiment 3: Statistical Tests}

Since each algorithm is run independently 50 times on each test function, and so on each test function, each algorithm has 50 samples, so this article uses the T test SAGBA algorithm are compared with those of the BA algorithm, SAPSO algorithm performance. The $\mathrm{T}$ test uses the degrees of freedom for 58, significant level of 0.05 two-tailed tests. The results show in Table 4. The inspection result "S +" said SAGBA algorithm on the bank of the calculation results to corresponding algorithm will be significantly better than that of the column, the column "S -" said of the algorithm on the bank of the significant better than SAGBA algorithm, " " said SAGBA algorithm on the bank of the performance and the column of the performance of the algorithm is the same. 
Table 4. $T$ Test of BAGBA Algorithm and the Other Two Kinds of Algorithm

\begin{tabular}{|c|c|c|}
\hline Test functions & $\mathrm{BA}$ & SAPSO \\
\hline Ackley & $\mathrm{S}+$ & $\mathrm{S}+$ \\
\hline Beale & $\mathrm{S}+$ & $\mathrm{S}+$ \\
\hline \multirow[t]{2}{*}{ Bohachevsky } & $\mathrm{S}+$ & $\mathrm{S}+$ \\
\hline & $\sim$ & S+ \\
\hline & $\mathrm{S}+$ & $\mathrm{S}+$ \\
\hline $\begin{array}{l}\text { Booth } \\
\text { Branin }\end{array}$ & $\mathrm{S}+$ & $\mathrm{S}+$ \\
\hline Dixon\&Price $(d=25)$ & $\mathrm{S}+$ & $\mathrm{S}+$ \\
\hline \multirow{2}{*}{$\begin{array}{l}\text { Goldstein\&Price } \\
\text { Griewank }\end{array}$} & S- & $\sim$ \\
\hline & $\sim$ & $\sim$ \\
\hline \multirow{2}{*}{ Hartmann } & $S_{1}$ & $C_{1}$ \\
\hline & $\mathrm{S}+$ & $\mathrm{S}+$ \\
\hline Levy & S+ & $\mathrm{S}+$ \\
\hline Matyas & $\sim$ & $\sim$ \\
\hline Powell( $d=24)$ & $\mathrm{S}+$ & $\mathrm{S}+$ \\
\hline & $\mathrm{S}_{+}$ & $\mathrm{S}+$ \\
\hline $\begin{array}{c}\text { Rosenbrock } \\
\text { Shubert }\end{array}$ & $\mathrm{S}+$ & $\mathrm{S}+$ \\
\hline \multirow{4}{*}{$\begin{array}{c}\text { Sphere }(\mathrm{d}=30) \\
\text { Sum Squares }(\mathrm{d}=20) \\
\text { Trid }(\mathrm{d}=6) \\
\text { Zakharov }\end{array}$} & $\sim$ & $\mathrm{S}+$ \\
\hline & $\mathrm{S}+$ & $\mathrm{S}+$ \\
\hline & $\mathrm{S}+$ & $\mathrm{S}+$ \\
\hline & $\mathrm{S}+$ & $\mathrm{S}+$ \\
\hline & $\sim$ & $\mathrm{S}+$ \\
\hline
\end{tabular}

From Table 4 can intuitively obvious, compared with the algorithm of BA, on Griewank function, significant difference in BA algorithm is better than SAGBA algorithm, the Booth function, Hartmann function and Matyas function, Shubert functions and Zakharov, SAGBA significance of our algorithm is the same as the BA algorithm, in addition to that, for the other functions, SAGBA algorithm is superior to the significance of BA. Compared with the SAPSO algorithm, in addition to the Griewank function, Hartmann functions and Matyas, SAGBA algorithm significance of the same with SAPSO algorithm, on the other function, SAGBA algorithm is significantly superior to SAPSO algorithm. We can draw the following conclusion: from the perspective of statistical SAGBA algorithm are significantly better than in most of the testing issue with BA SAPSO algorithm.

\section{(D) Experiment 4: Combinatorial Optimization Test}

Combinatorial optimization problem is to find the optimal variable combination problem under the given constraints, such as the work piece machining sequencing problem, vehicle routing problem, knapsack problem are typical combinatorial optimization problem, the problem has a strong engineering background and practical application value, but in the theory of computation is NP problem, optimization is very difficult to solve.

This article selects the replacement pipeline Scheduling Problem in the production Scheduling Problem (Permutation Flow - Shop Scheduling Problem, PFSP) test cases, the bat algorithm using Car class [9] Problem designed by Carlier as the test data, and compared with basic particle swarm algorithm. And in function optimization, the solution is different with problem can be directly from the individual position, application of the bat algorithm PFSP first need to adopt a reasonable coding way to represent the scheduling problem of the solution. Is adopted in this paper, according to the characteristics of PFSP random key encoding based on minimum location value rules 
[10], bat individuals will be a continuous position vector into the machine and a sequence of machining, to calculate the target individual corresponding scheduling solution. Through this transformation, it can not only guarantee the feasibility of the scheduling solution, then the need to modify the evolution operation of the bat algorithm. Algorithm of population number, the maximum number of $\operatorname{search}^{M a x T}=200$, the rest of the parameters set up day. Each algorithm independent running 20 times, test results as shown in Table 3.

In Table $5 \mathrm{P}$ represent problem type, $\mathrm{n}, \mathrm{m}$, respectively quantity and the machine number, $c^{*}$ according to the corresponding scheduling problem of minimizing the completion time. The SR (Success Rate) is the optimum Success Rate, on behalf of 20 times found in independent testing c "value ratio; BRE (Best Relative Error), motorcycle (Average Relative Error), WRE (Worst Relative Error) respectively the optimal, the Average Relative Error, the Worst.

Table 5. The Test Results of Combinatorial Optimization Problem

\begin{tabular}{|c|c|c|c|c|c|c|c|c|c|c|}
\hline \multirow{2}{*}{$\mathrm{P}$} & \multirow{2}{*}{$\mathrm{n}, \mathrm{m}$} & \multirow{2}{*}{$\mathrm{C}^{*}$} & \multicolumn{4}{|c|}{ SAGBA } & \multicolumn{4}{|c|}{ PSO } \\
\hline & & & BRE & ARE & WRE & SR & BRE & ARE & WRE & SR \\
\hline Car1 & 11,5 & 7038 & 0 & 0 & 0 & $100 \%$ & 0 & 0.07 & 1.53 & $95 \%$ \\
\hline Car2 & 13,4 & 7166 & 0 & 0.25 & 2.93 & $90 \%$ & 0 & 2.13 & 4.82 & $40 \%$ \\
\hline Car3 & 12,5 & 7312 & 0 & 0.71 & 3.16 & $50 \%$ & 0.87 & 2.74 & 4.18 & $0 \%$ \\
\hline Car4 & 14,4 & 8003 & 0 & 0 & 0 & $100 \%$ & 0 & 1.48 & 5.24 & $45 \%$ \\
\hline Car5 & 10,6 & 7720 & 0 & 1.02 & 1.65 & $15 \%$ & 0 & 1.65 & 4.27 & $10 \%$ \\
\hline Car6 & 8,9 & 8505 & 0 & 0.80 & 2.79 & $35 \%$ & 0 & 1.57 & 3.39 & $15 \%$ \\
\hline Car7 & 7,7 & 6590 & 0 & 0.24 & 0.80 & $80 \%$ & 0 & 0.42 & 2.55 & $75 \%$ \\
\hline Car8 & 8,8 & 8366 & 0 & 0.32 & 1.96 & $75 \%$ & 0 & 0.76 & 3.84 & $60 \%$ \\
\hline
\end{tabular}

As can be seen from Table 3 data, to the problem of the Car class test, SAGBA can found the known lower bound value, and optimization SAGBA success rate higher than the corresponding PSO algorithm, reflect SAGBA has good global convergence. From motorcycle and WRE data, scheduling scheme, is obtained by SAGBA, the average quality is far higher than that of PSO algorithm, because in the process of optimization and does not have the bat algorithm with any local search strategy, show SAGBA also has good evolutionary mechanism in discrete space, makes SAGBA also has a good application prospect in the field of combinatorial optimization.

\section{Conclusion}

This paper analyzes theoretically in the bat algorithm, and this algorithm absorbs the bat by ultrasound to search in the nature. The behavior of the predator prey characteristics, using the evolutionary approach to implement the agent's behavior in order to achieve the purpose of optimization. Simulated annealing algorithm combined with a bat optimization algorithm, to gauss perturbation of bats individuals, further search to retain the individual "elite". Along with the advancement of evolutionary process, the temperature is gradually reduced; accept the risk of poor solution gradually reduced, so as to improve the convergence of the algorithm. The convergence of the simulation results show that the proposed algorithm is superior to the other two algorithms in different degree, at the same time shows the SAGBA algorithms in continuous space and the feasibility and effectiveness of discrete space optimization, has a good application prospect. Because the bat algorithm optimization theory and application research is still in the initial stage, many problems has yet to be constantly explore and solve by people, such as involved in the algorithm of parameter Settings, the convergence of the algorithm analysis and the theoretical basis of the organic combination with the rest of the group of intelligent optimization algorithm, such as the study SAGBA algorithm compared with other 
optimization algorithms, and further research SAGBA algorithm in the application of multi-objective programming problems, these are the further research work to do.

\section{Acknowledgment}

This work was supported in part by the Natural Science Foundation of the Ningxia Hui Autonomous Region under grant no. NZ13048. The Humanities Arts and Social Sciences Fund of Higher Education of Tianjin under Grant No. 20102523.

\section{References}

[1] D. Karaboga, "An Idea Based on Honey Bee Swarm for Numerical Optimization", Technical Report-TR06, Kayseri: Erciyes University, (2005).

[2] D. Karaboga and B. Akay, "A Survey: Algorithms Simulating Bee Swarm Intelligence", Artificial Intelligence Review, vol. 31, no. 1-4, (2009), pp. 61-85.

[3] K. N. Krishnan and D. Ghose, "Glowworm Swarm Optimization: A New Method for Optimizing Multi-Modal Functions", International Journal of Computational Intelligence Studies, vol. 1, no. 1, (2009), pp. 93-119.

[4] X. S. Yang, "Nature Inspired Meta-heuristic Algorithms (2nd Edition)", Frome, UK: Luniver Press, (2010), pp. 97-104.

[5] Y. Shi and R. Eberhart, "A modified particle swarm optimizer", Proceedings of IEEE International Conference on Evolutionary Computation. Anchorage, USA: IEEE, (1998), pp. 69-73.

[6] M. F. Tasgetren, M. Sevkli, Y. C. Liang, et al., "Particle swarm optimization algorithm for permutation flow shop sequencing problem", Lecture Notes in Computer Science, vol. 3172, (2004), pp. 382-389.

[7] S. C. H. Leung, Z. De-Fu, Z. Chang-Le, et al., "A Hybrid Simulated Annealing Metaheuristic Algorithm for the Two-Dimensional Knapsack Packing Problem", Computed Operation Research, vol. 39, no. 1, (2012), pp. 64-73.

[8] D. Simon, "Biogeography-Based Optimization", IEEE Transactions on Evolutionary Computation, vol. 12, no. 6, (2008), pp. 702-713.

[9] X. S. Yang, "A new metaheuristic bat-inspired algorithm", Nature Inspired Cooperative Strategies for Optimization (NICSO 2010), Studies in Computational Intelligence, Springer-Verlag, Berlin Eidelberg, vol. 284, (2010), pp. 65-74.

[10] X. S. Yang, "Bat Algorithm for Multi-objective Optimization”, Int. J. Bio-Inspired Computation, vol. 3, no. 5, (2011), pp. 267-274.

[11] T. A. Lemma, "Use of fuzzy systems and bat algorithm for energy modeling in a gas turbine generator", Science and Engineering, (2011), pp. 305-310.

[12] X. S. Yang and A. H. Gandomi, "Bat algorithm: a novel approach for global engineering optimization", Engineering Computations, vol. 29, no. 5, (2012), pp. 464-483.

[13] S. Mishra, K. Shaw and D. Mishra, "A new metaheuristic classification approach for microarray data", Procedia Technology, vol. 4, no. 1, (2012), pp. 802-806.

[14] K. Khan, A. Nikov and A. Sahai, "A Fuzzy Bat Clustering Method for Ergonomic Screening of Office Workplaces", S3T Advances in Intelligent and Soft Computing, vol. 101, (2011), pp. 59-66.

[15] Z. Lv, L. Feng, H. Li and S. Feng, "Hand-free motion interaction on Google Glass", In SIGGRAPH Asia 2014 Mobile Graphics and Interactive Applications, ACM, (2014), pp. 21.

[16] C. Zhong, S. M. Arisona, X. Huang, M. Batty and G. Schmitt, "Detecting the dynamics of urban structure through spatial network analysis", International Journal of Geographical Information Science, vol. 28 , no. $11,(\mathbf{2 0 1 4})$, pp. $2178-2199$.

[17] Z. Lv and T. Su, "3D seabed modeling and visualization on ubiquitous context", In SIGGRAPH Asia Posters, ACM, (2014), pp. 33.

[18] Z. Lv, L. Feng, S. Feng and H. Li, "Extending Touch-less Interaction on Vision Based Wearable Device", Virtual Reality (VR), IEEE, (2015).

[19] S. Li, Y. Geng, J. He and K. Pahlavan, "Analysis of Three-dimensional Maximum Likelihood Algorithm for Capsule Endoscopy Localization", 5th International Conference on Biomedical Engineering and Informatics (BMEI), Chongqing, China, (2012) October, pp. 721-725.

[20] Y. Geng, J. He, H. Deng and K. Pahlavan, "Modeling the Effect of Human Body on TOA Ranging for Indoor Human Tracking with Wrist Mounted Sensor", 16th International Symposium on Wireless Personal Multimedia Communications (WPMC), Atlantic City, NJ, (2013) June. 


\section{Authors}

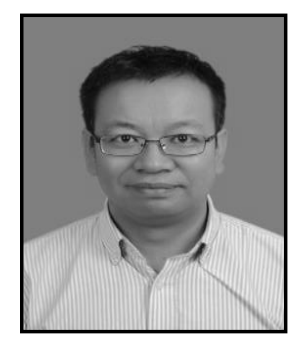

Guodong Zhao, was born in Ningxia, China, 1972. He received the B.Sc. degree from Ningxia University, China, in 1994, the M.Edu. degree from Ningxia University, China, in 2007. He is currently working toward a Ph.D. degree in Tianjin Polytechnic University, China. He was with the School of Mathematics and Computer science, Ningxia University since1994, as an associate professor. His research interests include high performance embedded system, fault tolerant algorithms and architectures.

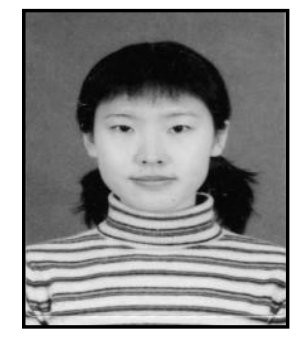

Ying Zhou, received the B.Sc. degree from Nankai University, China, in 2002, the M. Eng. degree from Nankai University, China, in 2005. Currently, she is a Ph.D. candidate in Tianjin Polytechnic University, China. She was with the school of science and Engineering, Tianjin Open University since 2005, as an associate professor. Her research interests include high performance embedded system, fault tolerant algorithms and architectures. 